\title{
SDU Discard Function of RLC Protocol in UMTS
}

\author{
Vit Krichl ${ }^{1}$ \\ ${ }^{1}$ Czech Technical University in Prague, Faculty of Electrical Engineering, Department of \\ Telecommunication Engineering, Technicka 2, 16627 Prague, Czech Republic \\ krichv1@fel.cvut.cz.
}

\begin{abstract}
This paper deals with Radio Link Control protocol (RLC) in UMTS mobile network. It describes in brief the RLC protocol, its functions, properties, parameters, data structure and functional modes. In detail this study reports the acknowledged mode SDU discard procedure and its effect on the protocol performance. For the purpose of monitoring behavior of two RLC entities and performance evaluation of the RLC protocol with different values of RLC parameters a simulation program was developed. Graphic simulation outputs reporting the effect on performance are attached in the paper.
\end{abstract}

Keywords: RLC, UMTS, performance, Radio Link Control, SDU discard

\section{Introduction}

UMTS (Universal Mobile Telecommunication System) is a standard of the $3^{\text {rd }}$ generation of mobile telecommunication systems (3G) under specification of 3GPP organization ( $3^{\text {rd }}$ Generation Partnership Project). WCDMA (Wideband Code Division Multiple Access) on the radio link interface, spread spectrum and high data rates (above $1 \mathrm{Mbps}$ ) are the significant features of this technology.

\subsection{UMTS Architecture}

The architecture of UMTS network is very similar to the $2 \mathrm{G}$ (GSM) network architecture. The network elements can be divided in two groups. Radio Access Network - RAN (often called UTRAN - UMTS Terrestrial RAN) provides all the radio service and the access to the core network for the mobile user via radio interface. Analogy in GSM is the BSS (Base Station Subsystem). Core Network CN is responsible for switching and routing functions of voice and data calls and cooperation with external circuit switched (CS) and packet switched (PS) networks. Analogy in GSM is NSS (Network Switching Subsystem). The last part of the UMTS network are the User Equipments (UE) such as mobile phones or laptops. In the UMTS architecture many interfaces ( $\mathrm{Iu}, \mathrm{Uu}$...) are also defined and standardized. 


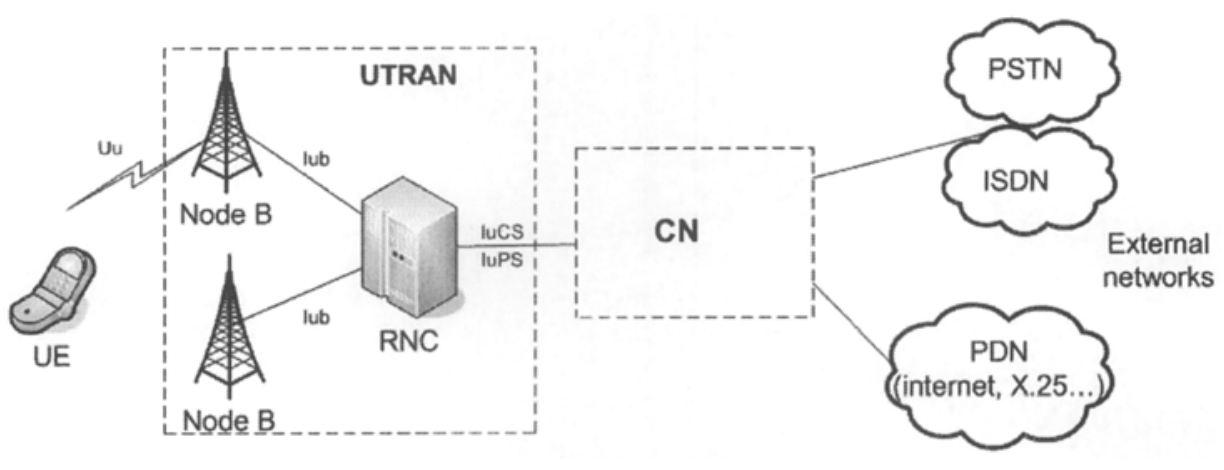

Fig. 1. General UMTS architecture ([1]).

\subsection{UTRAN Protocol Architecture}

Fig. 2 shows the UTRA interface protocol architecture and relation to the OSI reference model. The data link layer (L2) is divided in the control plane into MAC (Medium Access Control) and RLC (Radio Link Control) protocol layer. In the user plane there are 2 more service-dependent protocols: PDCP (Packet Data Convergence Protocol) for header compression and BMC (Broadcast/ Multicast Control Protocol). In the control plane of network layer (L3) is RRC (Radio Resource Control).

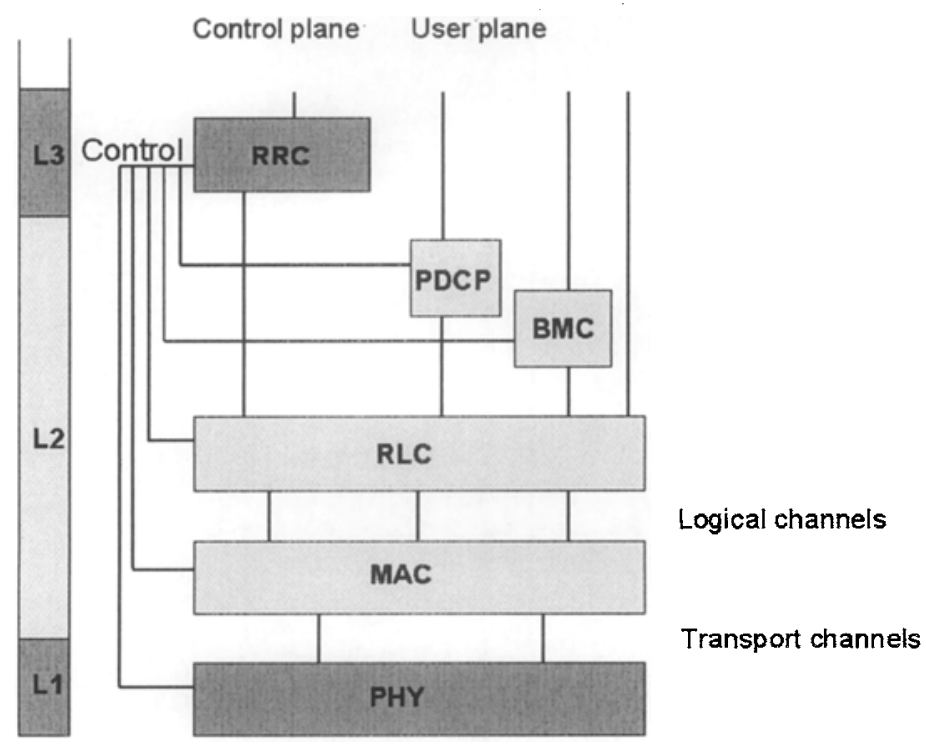

Fig. 2. UTRA protocol architecture ([2]). 


\section{Radio Link Control Protocol}

RLC (Radio Link Control) protocol provides the functionality of the data link layer on the WCDMA radio interface in the UE (user end) and in the RNC (network end). It is responsible for the correct data blocks delivery. It involves the configuration of the radio link parameters and reconfiguration in case of condition change. It works simultaneously in the user and control plane and provides the services for the CS and PS links. RLC protocol provides services for user even for control data. Segmentation, reassembly, concatenation of data blocks, padding, error correction, insequence delivery of upper-layer data blocks, duplicate detection, flow control, sequence number check, ciphering is example of protocol layer functions.

\subsection{RLC Data Structure}

RLC protocol receives upper layer data packets called RLC SDU (RLC Service Data Units). For ensure the requested functions and cooperation with the lower layer (MAC), RLC protocol uses its own data structure by changing format, length and header of the SDU. These protocol specific blocks are called RLC PDU (Protocol Data Units). There are two types of RLC PDUs. Data PDU is used for upper-layer user data (RLC Service Data Unit, RLC SDU) transport and is defined for every functional mode. Control PDUs are used only in Acknowledged Mode for indication of correctly received/corrupted/ missing PDUs and changing values of protocol parameters.

\subsection{RLC Modes}

The upper-layer protocol RRC (Radio Resource Control) chooses one of three possible functional RLC modes.

Transparent Mode (TM) adds to the upper-layer data no header. The Receiver simply deletes or marks the erroneous data blocks. TM can be used for voice services, video-telephony or multimedia applications with stream data transfer.

Unacknowledged Mode (UM) uses the sequence numbering of data blocks (PDU) but has no implemented mechanism for their retransmission that's why the data blocks delivery can not be guaranteed. Unacknowledged mode is suitable for Voice over IP (VoIP) and cell broadcast services.

Acknowledged Mode (AM) is using sequence numbering of data blocks and sophisticated retransmission mechanism (requires backwards channel for acknowledgements) for correct user data delivery. The RLC AM is usually used in packet services such as FTP data traffic, or web browsing. Only acknowledged mode network entities will be assumed considered in the next parts of this paper. 

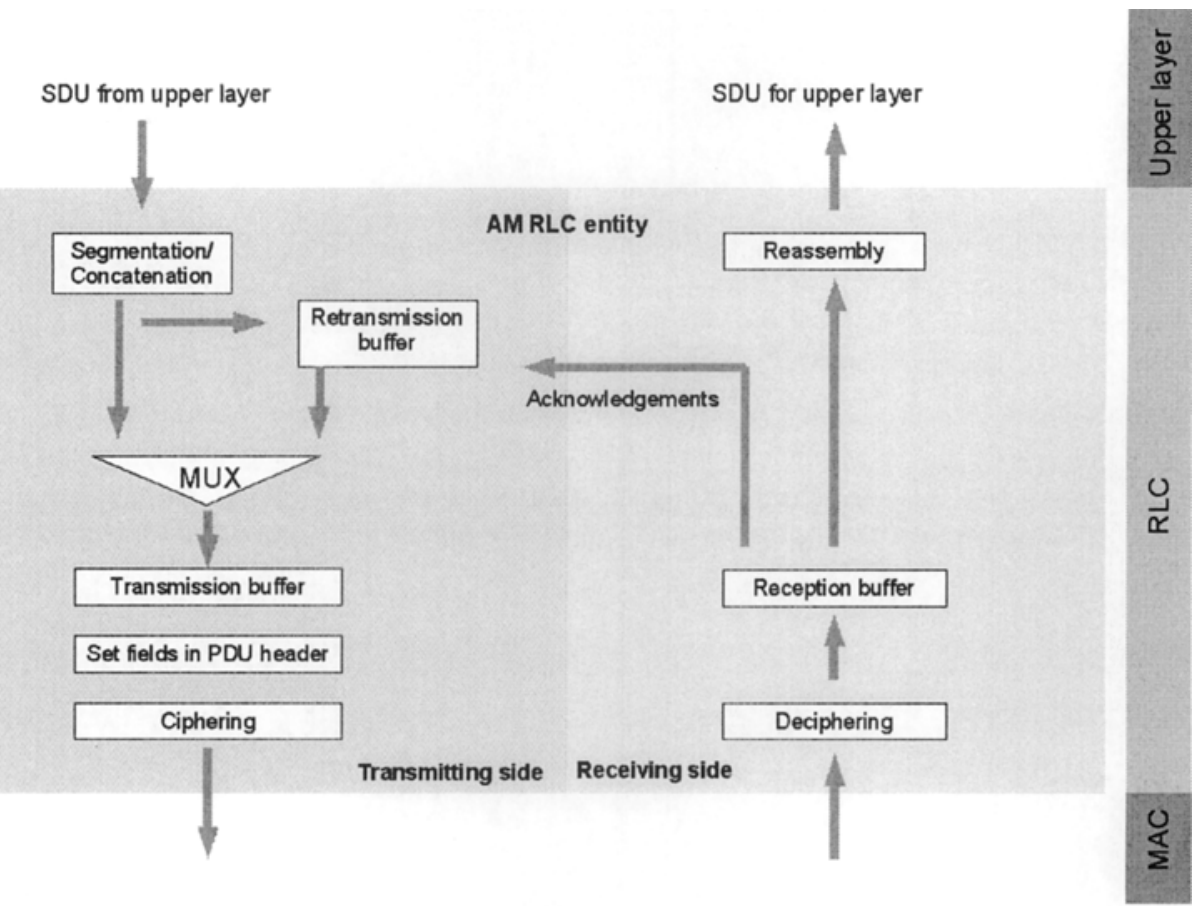

Fig. 3. Acknowledged mode RLC entity model ([3]).

Error correction in the AM is provided by the selective ARQ (Automatic Repeat Request) mechanism. The receiver acknowledges the correctly received PDUs. If erroneous PDU is detected, the receiver requires repeated transmission of this PDU. For effective data transfer a transmitting window is used, it is not necessary to acknowledge every single PDU. On the other side it is not effective to repeat the PDU transmission many times. Many attempts of transfer 1 PDU can delay other data blocks. That's why a SDU discard function is used.

\section{SDU Discard Function}

The SDU discard function is used by the Sender to discharge RLC PDUs from the RLC PDU buffer. If the transmission of single PDU with a given sequence number does not succeed in defined time or in defined number of transmissions, this PDU and all other PDUs belonging to the same SDU are deleted and no more indicated for transmission. SDU discard function allows avoid buffer overflow. There are several alternative operation modes of the RLC SDU discard function. For an acknowledged mode RLC entity, one of these modes is always configured by upper layers control:

- SDU discard after limited number of transmissions

- Timer based discard with explicit signaling

- No discard after limited number of transmissions 


\subsection{SDU Discard after Limited Number of Transmissions}

Every PDU scheduled for transmissions increases its counter in the Sender. If the counter reaches a given threshold, the relevant SDU will be discarded. The Receiver is notified about discarded SDU by the Status PDU message directly from the Sender. This form of SDU discard function is dependent on the channel rate and its changes and it can not guarantee the maximum delay value. On the other hand it strives to keep the SDU loss rate constant for the connection.

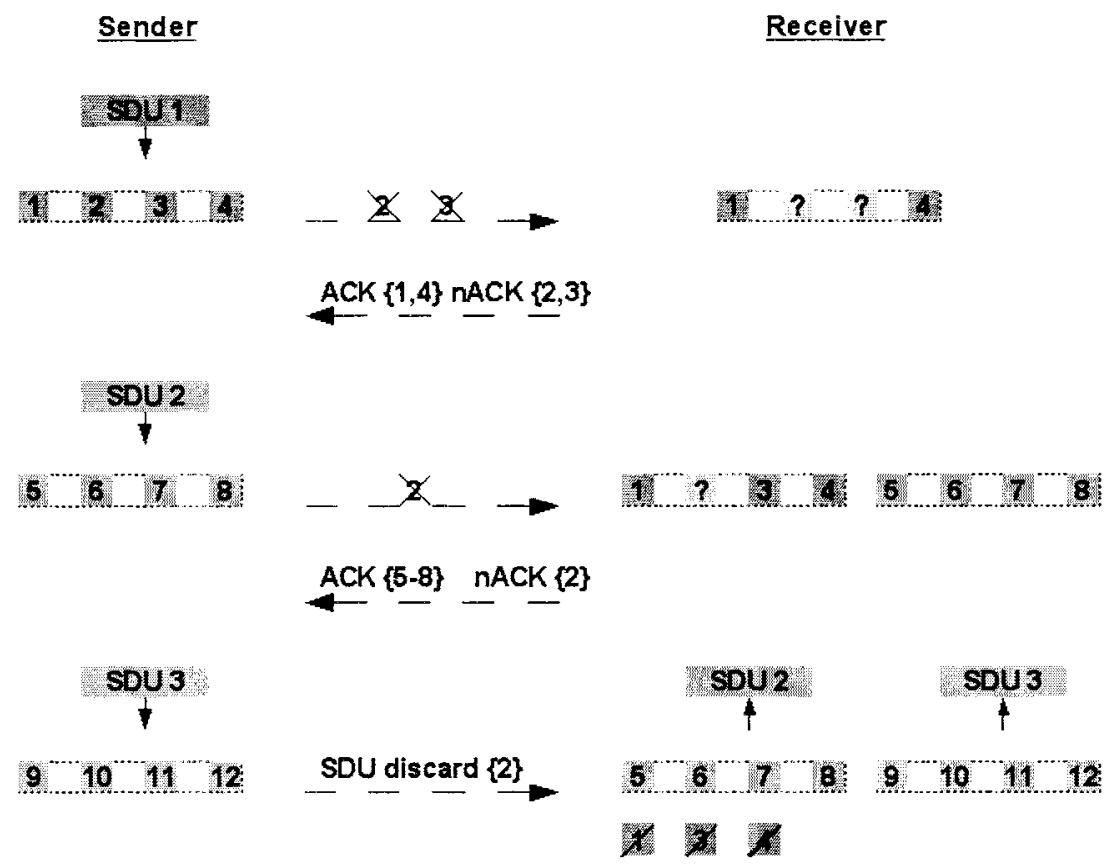

Fig. 4. Simplified SDU discard procedure with 1 retransmission allowed.

For an example of SDU discard procedure (configured with one possible retransmission) see Fig. 5. The first incoming data block SDU 1 is segmented in the RLC sublayer into 4 RLC PDUs. During the transmission of these data blocks PDU 2 and PDU 3 were corrupted that is reported by the Receiver in Status PDU. The Sender will transmit these specified PDUs again together with other (new) segmented PDUs. Because of In-sequence delivery configuration, the Receiver can not submit the reassembled SDU 2 to the upper layer before reception of all PDUs of SDU 1. After reception of the second Status PDU, the Sender does not submit PDU 3 for transmission, because of reached limited number of retransmissions. All fragments of SDU 1 are deleted from (re)transmission buffer and Status PDU is generated to inform the Receiver (move the reception window). 


\subsection{Timer Based SDU Discard with Explicit Signaling}

This version uses a timer based triggering SDU discard. For every SDU received from upper layers, the Sender starts a discard timer. When the discard timer of a SDU expires, the Sender will discard the appropriate SDU and inform the Receiver by a Status PDU (including Move reception window command). This SDU discard mode is insensitive to variations in the channel rate and provides means for exact definition of maximum delay. However, the SDU loss rate of the connection is increased as SDUs are discarded. Possible values of discard timer parameter are specified in [4].

\subsection{No Discard after Limited Number of Transmissions}

In this alternative mode, the RLC reset procedure is initiated by the Sender after reaching the limited number of PDU transmissions. This version of SDU discard function is not further more described in this paper.

\section{Simulation}

The created model evaluates behavior of two RLC entities (UE and RNC), their data PDU and control PDU exchange. It provides mainly RLC functions such as segmentation and reassembly of upper layer data blocks, padding of PDU, data PDU and control PDU transfer, ARQ mechanism for error correction and in-sequence delivery of upper-layer data blocks. For better understanding of the RLC parameters influence, the backward channel (for acknowledgments) is considered error-free. In this part of simulation the input data rate was considered constant-size SDU stream with constant time intervals between SDUs. This concept could be simplified model of FTP traffic. For simulating the radio channel conditions - error rates, the Gilbert model was chosen for its easy implementation (often used in wireless networks models). Assigning the probability values was based on data frame trace analysis of GSM radio link with CSD (Circuit Switched Data) service [5]. Modifying these values, we can simulate the radio channel conditions with different BLER.

Table 1. Simulation parameters default values.

\begin{tabular}{|l|l|}
\hline Simulation parameter & Parameter value \\
\hline Transmission time interval & $10 \mathrm{~ms}$ \\
\hline SDU size & 1280 bytes \\
\hline SDU data rate & $512 \mathrm{kbps}$ \\
\hline PDU size & $80+2$ header bytes \\
\hline Status timer period & $100 \mathrm{~ms}$ \\
\hline PDU per TTI & max 16 \\
\hline PDU one-way delay & $45 \mathrm{~ms}$ \\
\hline Window Size & 256 PDUs \\
\hline
\end{tabular}


Table 1 shows the default simulation parameters values. Every incoming SDU of 1280 bytes is first segmented. During one transmission time interval $10 \mathrm{~ms}$ up to 16 PDUs each of 82 bytes can be sent. The Receiver generates every $100 \mathrm{~ms}$ a Status PDU to acknowledge the correct received PDUs and to report the corrupted PDUs. The average time interval between submitting PDU for the lower layer in RNC (network side) and PDU processing in UE (user side) is fixed $45 \mathrm{~ms}$. This time interval includes one TTI, network propagation time and delays caused by a queue of other traffic PDUs in Node B. For performance evaluation and parameter values effect estimation we simulate $5000 \mathrm{~ms}$ of network traffic.

\section{Simulation Results}

\subsection{Limited Number of Retransmission Effect}

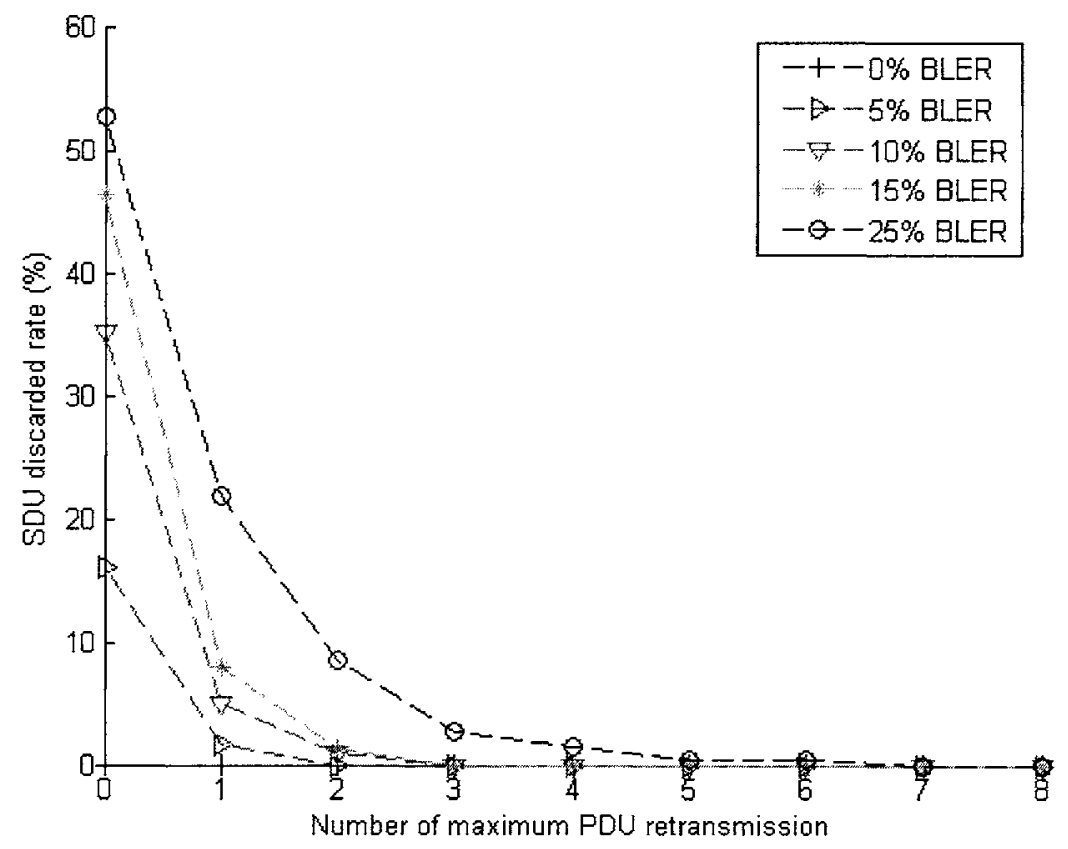

Fig. 5. Effect on SDU discarded rate with different BLER.

The Fig. 4 shows that the portion of SDU discarded during data transmission rapidly increase with more possible attempts for transmission. With 2 retransmissions the share of discarded SDU is lower than $10 \%$ in all simulated error rates. Radio channel with BLER $<15 \%$ has this share under $10 \%$ with only one retransmission. Configuring even higher number of retransmission causes only small decrease of the SDU discarded share. High number of possible retransmissions causes rapid increase 
of RLC SDU's delay due to requested in-sequence delivery of reassembled RLC SDU to upper layer, transmission buffer queuing delay. The SDU delay is also influenced by the status PDUs interval (time period elapsed between 2 control PDUs reporting the corrupted PDUs). Marginally, the transmitting window can be blocked by the PDUs for retransmission.

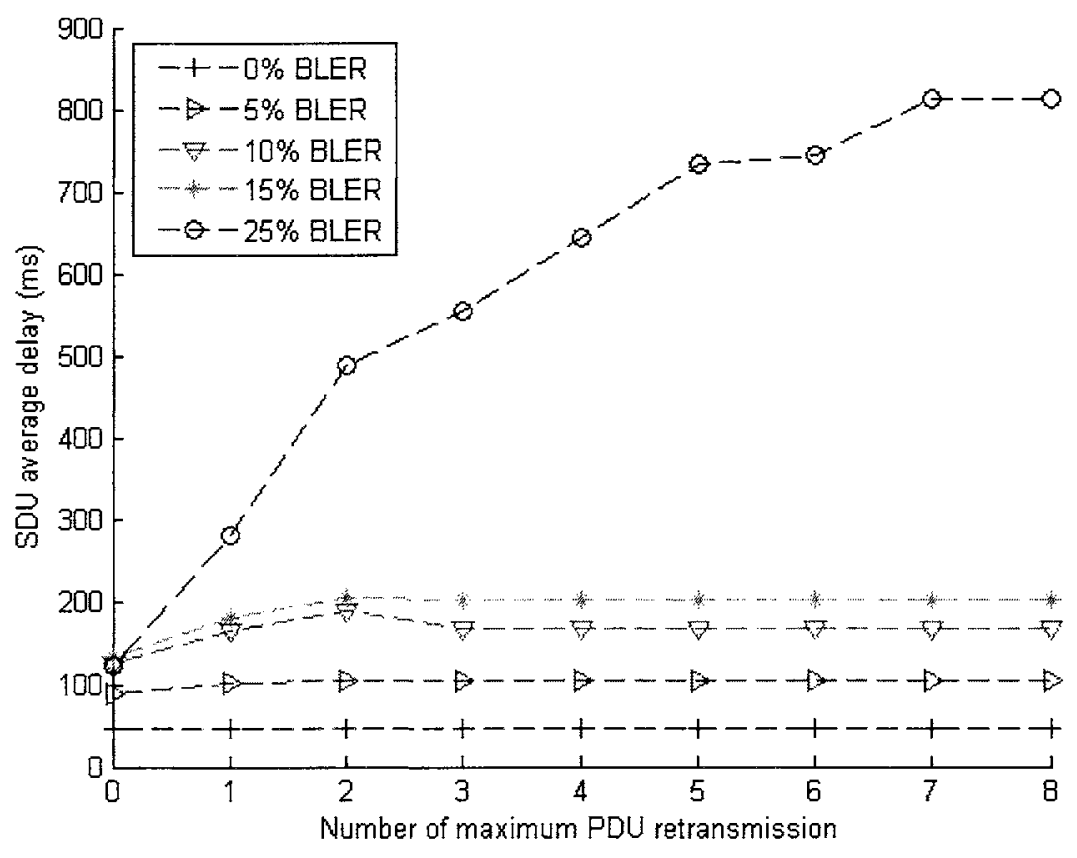

Fig. 6. Effect on SDU average delay with different BLER.

Because of increasing SDU delay it is not suitable to configure the RLC link (with configuration given above) with more than 4 attempts at RLC SDU delivery. The RLC link on the radio channel with low level error rate can be even configured with only 1 or 2 retransmission attempts. In case of RLC PDU transport failure even with reaching the limit of retransmission, an additional error correction of this RLC SDU transfer can be performed by higher layer protocols such as TCP (Transmission Control Protocol) in transport layer. 


\subsection{Discard Timer Value Effect}

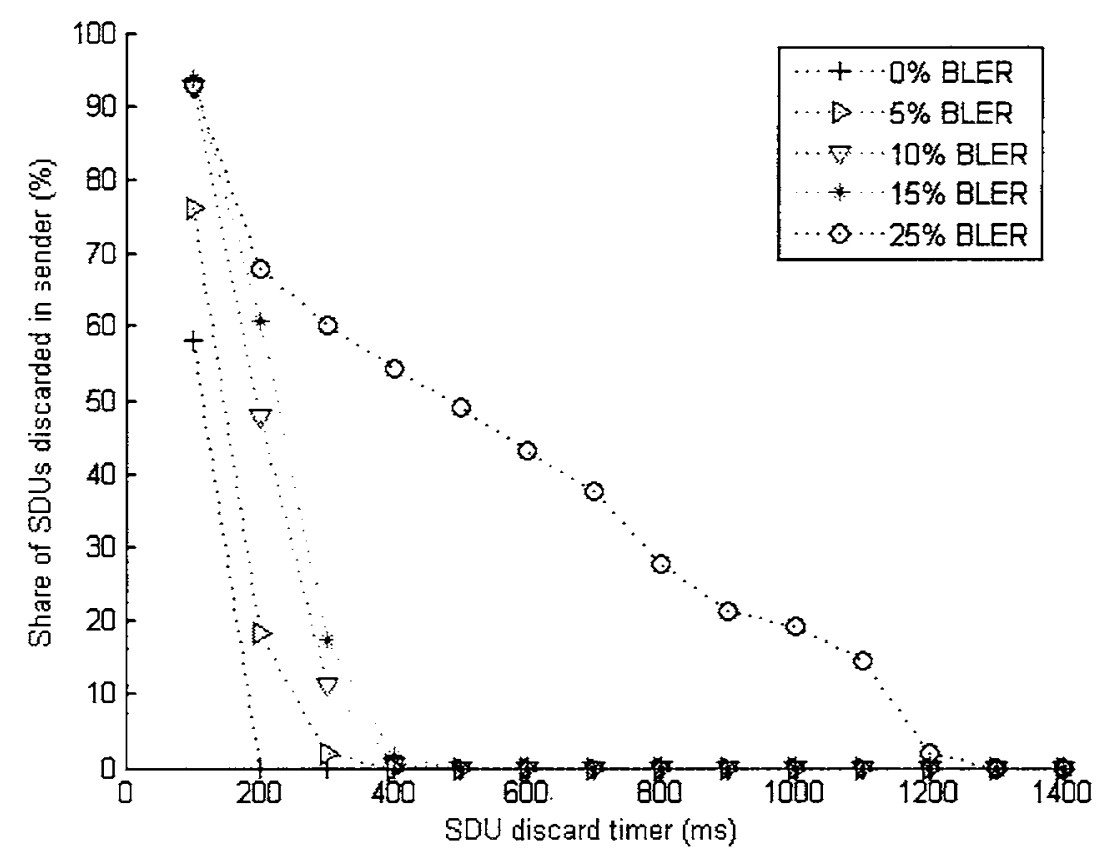

Fig. 7. SDU discard ratio in Sender.

Even with error-free radio channel, with $100 \mathrm{~ms}$ of discard timer, there is non-zero SDU discard share because the PDUs are deleted in Sender before Status PDU acknowledges a correct PDU delivery. On the radio channel with block error-rate less than $20 \%$, it is not necessary to configure the RLC link with SDU discard timer 300 ms or more - leads only to higher SDU delay, SDU discard share is lower than $20 \%$. In conditions of higher error rates, this function provides transmission buffer overflow protection, a significant part of the buffer memory (more than $30 \%$ ) is periodically cleaned. The number of discarded SDU is also influenced by the time interval between Status PDU reports. Mainly with lower values of discard timer, the portion of SDU discarded in Sender is really high, but it does not mean necessarily SDU loss. As we can see (Fig. 8) SDU discard ratio in Receiver is lower. SDU discard is triggered by the elapsed discard timer in Sender, all PDUs belonging to the SDU are deleted from (re)transmission buffer and a Status PDU report is generated. Before this Status PDU (reporting SDU discard) reaches the Receiver, all relative PDUs sent in previous TTIs could be completed and SDU could be reassembled. Reception of Status PDU in Receiver then only move reception window (no deletion of PDUs from receiving buffer). 


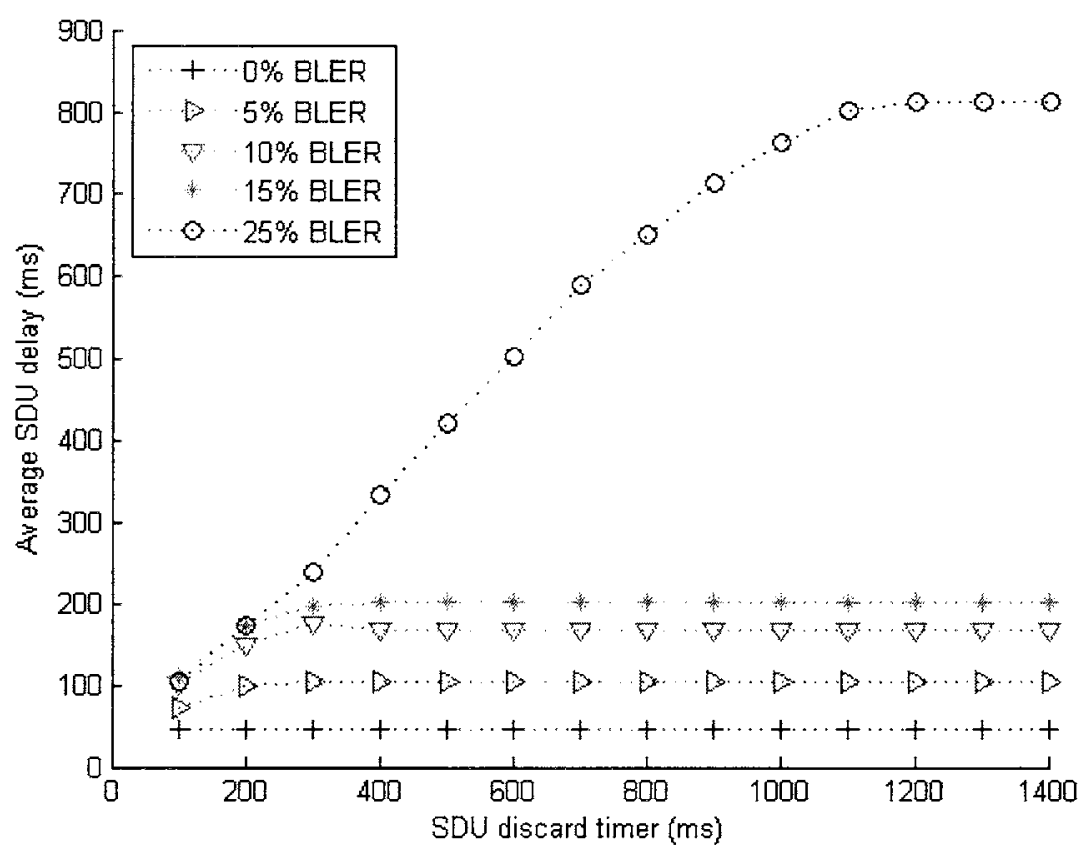

Fig. 8. SDU delay with variable discard timer value.

As a consequence of bigger discard timer value and in-sequence delivery configured in the Receiver, the delay of SDUs is increasing (Fig. 8.). First, the delay increases as a linear function of the discard timer value, the second part of the curve is constant delay line. Thanks to discard timer it can not rise more, the delay is saturated. This is typical feature of this discard function version, which can be used for Quality of Service (maximum delay) guarantee. In theory, maximum possible delay can be determined as a sum of discard timer value and PDU one-way delay, in case of blocked transmission window (very high error rate, too small transmission window). 


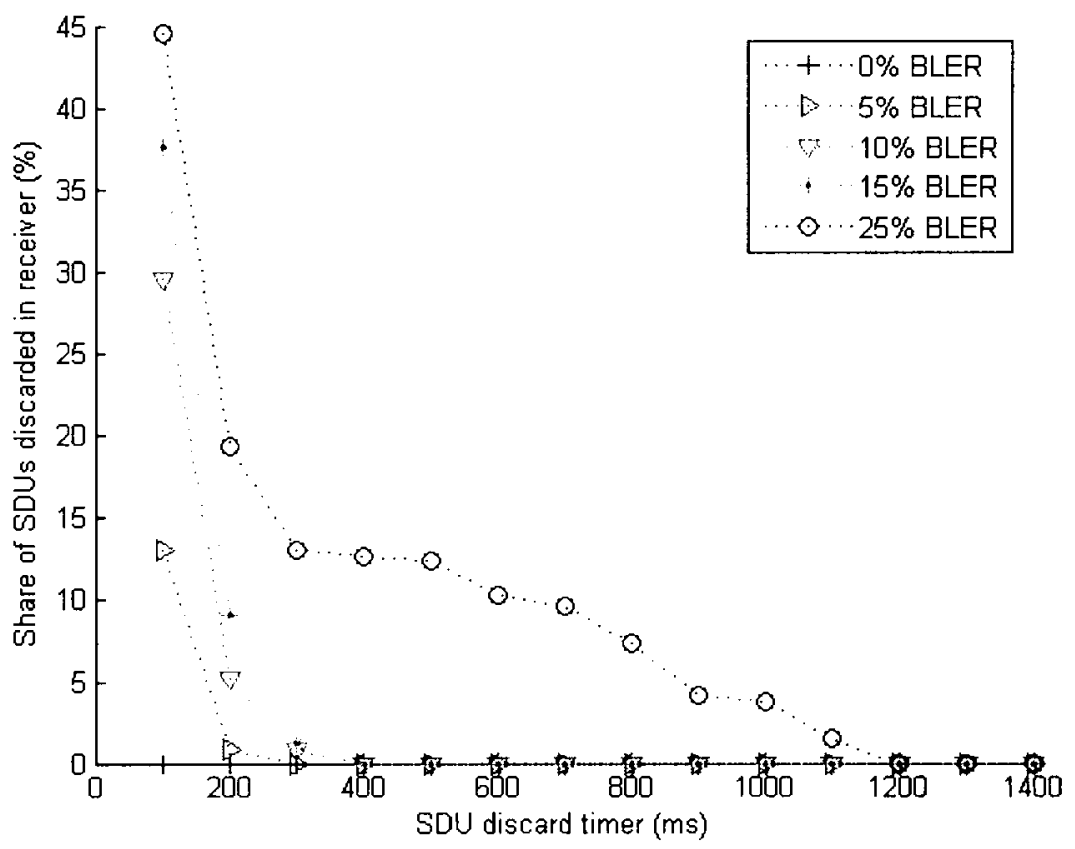

Fig. 9. SDU discard ratio in Receiver.

\section{Conclusion}

RLC protocol is effective mechanism supporting reliable connection and user data transfers on radio interface in UMTS. Some performance evaluation of RLC protocol concerning mainly the polling mechanism and Status PDU report interval was published in the past [6]. Effect of SDU discard function can be found in our study, which has only evaluated an effect of SDU discard triggers on the RLC performance (considered independently of the other PDU retransmissions triggers, but there are more triggers that can invoke transmission of a status report). The future studies can focus on the optimization of two or more triggers which are simultaneously active. The model developed in Matlab environment is easily to extend or improve.

Our simulation results indicate that the RLC protocol with number of retransmissions triggered SDU discard function can have the optimal performance with given configuration when the number of retransmission is set to 1 or 2 . With higher number of possible PDU retransmission, the SDU discarded rate is low, but the SDU delay is extremely high and for many required services unacceptable. Timer triggered SDU discard function provide means of guarantee QoS, data blocks delay in detail. The more intensive data rate, the more SDU discarded ratio because of higher 
probability of transmission window blocked and heavy retransmission. When no PDU retransmission is possible (because of discard timer, PDU transmission counter) PDUs would be discarded and the higher-layer protocol (i.e. TCP) would have to provide correct delivery of these data blocks.

Acknowledgments. This research work was supported by Czech Technical University's grant No. CTU0715013.

\section{References}

1. Kaaranen, H.: UMTS networks: architecture, mobility and services, $2^{\text {nd }}$ edition, Chichester: Wiley (2005) ISBN 0-470-01103-3

2. Holma, H., Toskala, A.: WCDMA for UMTS: Radio Access for Third Generation Mobile Communication, $3^{\text {rd }}$ edition. Chichester : Wiley (2004) ISBN 0-470-87096-6.

3. Radio Link Control (RLC) protocol specification (Release 7), 3GPP TS 25.322 V7.2.0 (2006-10). http://www.3gpp.org/ftp/Specs/html-info/25322.htm

4. Radio Resource Control (RRC) specification (Release 7): 3GPP TS 25.331 V7.2.0 (2006-09) http://www.3gpp.org/ftp/Specs/html-info/25331.htm

5. Konrad, A., Zhao, B.: A Markov-Based Channel Model Algorithm for Wireless Network, ACM Wireless Networks (2003) http://bnrg.cs.berkeley.edu/ adj/publications/paper-files/

6. Li, J., Montuno, D., Wang, J.: Performance Evaluation of the Radio Link Control Protocol in 3G UMTS, MITACS (2004)

http://www.scs.carleton.ca/ canccom/Publications/LiPerfEvalRLC04.pdf 